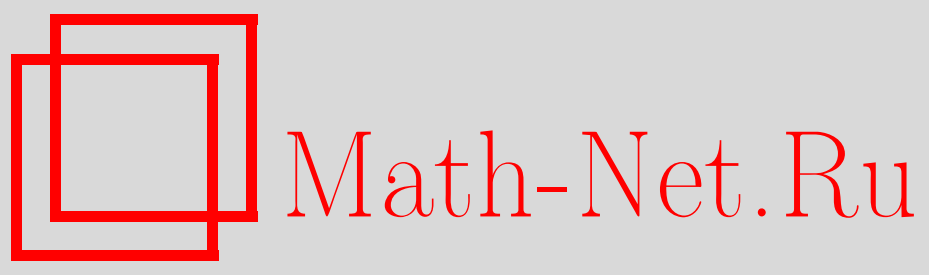

М. Б. Хрипунова, А. А. Юдин, Оценка функции концентрации для одного класса аддитивных функций, $M a$ тем. заметки, 2007, том 82, выпуск 4, 598-605

DOI: https://doi.org/10.4213/mzm3857

Использование Общероссийского математического портала Math-Net.Ru подразумевает, что вы прочитали и согласны с пользовательским соглашением http://www. mathnet.ru/rus/agreement

Параметры загрузки:

IP : 44.207 .124 .84

26 апреля 2023 г., 17:30:35

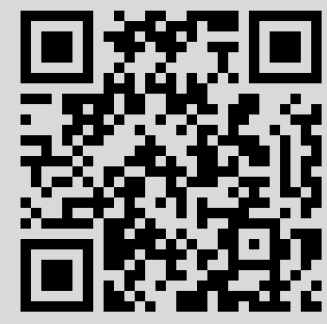


Том 82 выпуск 4 октябрь 2007

УДК 511

\section{Оценка функции концентрации для одного класса аддитивных функций}

\section{М. Б. Хрипунова, А. А. Юдин}

В работе показано, как методы оценки функции концентрации суммы независимых случайных величин дают возможность получать оценки функции концентрации для значений аддитивных функций при подходящих условиях. Ранее полученные результаты оценки функции концентрации являются следствиями оценки, полученной в настоящей заметке для функций из рассматриваемого класса.

Библиография: 10 названий.

Введение и формулировка основного результата. Обозначим через $f(n)$ арифметическую аддитивную функцию. Таким образом, для любого натурального $n$ функция $f(n)$ принимает целые рациональные значения и для любой пары натуральных взаимнопростых чисел $n_{1}$ и $n_{2}, f\left(n_{1} \cdot n_{2}\right)=f\left(n_{1}\right)+f\left(n_{2}\right)$. Определим класс $S_{\alpha}$. Арифметическая аддитивная функция $f(n)$ принадлежит классу $S_{\alpha}$, если найдутся такие величины $\tau_{a}$, что при каждом целом $a$ выполняется равенство

$$
\frac{1}{\pi(x)} \sum_{\substack{p \leqslant x \\ f(p)=a}} 1=\tau_{a}+\frac{\varepsilon_{x}(a)}{(\log x)^{\alpha}},
$$

где $\alpha>1, c$ не зависит от $x$ и от $a, \sum_{a}\left|\varepsilon_{x}(a)\right|<c<+\infty$ для любого $x, \pi(x)$ - число простых не превосходящих $x$ и $\tau_{0}<1$.

Пусть

$$
Q(x, f)=\max _{a} \sum_{\substack{f(n)=a \\ n \leqslant x}} 1
$$

- функция концентрации арифметической аддитивной функции $f(n)$.

Сформулируем основной результат.

TeOpema 1. Пусть $f(n) \in S_{\alpha}$, mozдa

$$
Q(x, f) \ll \frac{\max _{k} \tau_{k}}{\sqrt{1-\tau_{0}}} \frac{x}{(\log \log x)^{1 / 2}} .
$$

Работа выполнена при поддержке Российского фонда фундаментальных исследований (грант № 05-01-00617). 
ЗАмечание 1 . Отметим, что результат теоремы 1 дает возможность учесть влияние арифметической структуры множества значений $f(n)$ при оценке функции концентрации.

ЗАмечАниЕ 2. Покажем, что оценка, полученная в [1],

$$
Q(x, f) \ll x(E(x))^{-1 / 2}, \quad \text { где } \quad E(x)=\sum_{\substack{f(p) \neq 0 \\ p \leqslant x}} \frac{1}{p},
$$

является следствием теоремы 1. В самом деле, в условиях теоремы 1

$$
E(x)=\sum_{p \leqslant x} \frac{1}{p}-\sum_{\substack{f(p)=0 \\ p \leqslant x}} \frac{1}{p} \geqslant \log \log x-\tau_{0} \log \log x=\left(1-\tau_{0}\right) \log \log x
$$

и тогда оценку (1) можно записать так:

$$
Q(x, f) \ll x\left(\log \log x-\tau_{0} \log \log x\right)^{-1 / 2}=\frac{x}{\sqrt{1-\tau_{0}}}(\log \log x)^{-1 / 2} .
$$

Точно такая же оценка следует из нашей теоремы 1.

ЗАмЕчАниЕ 3. Оценка, полученная в [2], для функции концентрации арифметической аддитивной функции такова:

$$
Q(x, f) \ll x\left[\min _{\lambda}\left(\lambda^{2}+\sum_{p \leqslant x} \min \frac{1}{p}\left(1,(f(p)-\lambda \log p)^{2}\right)\right)^{-1 / 2}\right] .
$$

Если $f(n) \in S_{\alpha}$, то $\min \left(1,(f(p)-\lambda \log p)^{2}\right) \leqslant 1$ и, полагая $\lambda=0$, получаем, что указанная оценка в этом случае не лучше, чем

$$
Q(x, f) \leqslant x(\log \log x)^{-1 / 2} .
$$

Доказательство теоремы 1. Далее будем использовать теоретико-числовые методы работы [3] и современные методы оценки функции концентрации из работы [4]. Функцию концентрации арифметической аддитивной функции $f(n)$ запишем в виде интеграла

$$
Q(x, f)=\int_{0}^{1}\left(\sum_{n \leqslant x} e^{2 \pi i \xi f(n)}\right) \cdot e^{-2 \pi i \xi a} d \xi
$$

По лемме 2 из [3]

$$
\sum_{n \leqslant x} e^{2 \pi i \xi f(n)}=a(\xi) \cdot x \cdot(\log x)^{\tau(\xi)-1}+O\left(\frac{x}{\log x}\right)
$$

равномерно по $\xi \in \mathbb{R} / \mathbb{Z}=\mathbb{T}$,

$$
a(\xi)=\frac{1}{\Gamma(\tau(\xi))} \prod_{p}\left(1+\sum_{r=1}^{\infty} \frac{e^{2 \pi i \xi f\left(p^{r}\right)}}{p^{r}}\right)\left(1-\frac{1}{p}\right)^{\tau(\xi)},
$$


где $\Gamma(s)$ - гамма-функция Эйлера,

$$
\tau(\xi)=\sum_{k \in \mathbb{Z}} \tau_{k} e^{2 \pi i k \xi}, \quad \tau_{k} \geqslant 0 \quad \forall k, \quad \sum_{k \in \mathbb{Z}} \tau_{k}=1 .
$$

Из (2) и (3) получаем, что

$$
\begin{aligned}
\frac{Q(x, f)}{x} & =\max _{h \in \mathbb{Z}} \int_{0}^{1}\left[a(\xi)(\log x)^{\tau(\xi)-1}+O\left(\frac{1}{\log x}\right)\right] \cdot e^{-2 \pi i \xi h} d \xi \\
& \leqslant \max _{h \in \mathbb{Z}}\left|\int_{0}^{1}\left(a(\xi)(\log x)^{\tau(\xi)-1}+O\left(\frac{1}{\log x}\right)\right) \cdot e^{-2 \pi i \xi h} d \xi\right| \\
& \leqslant \max _{h \in \mathbb{Z}}\left|\int_{0}^{1} a(\xi)(\log x)^{\tau(\xi)-1} e^{-2 \pi i \xi h} d \xi\right|+\frac{c}{\log x},
\end{aligned}
$$

где $c$ - абсолютная постоянная.

Так как $a(\xi)$ - непрерывная на $[0,1]$ функция, то $a(\xi)$ ограничена на $[0,1]$. Следовательно,

$$
\begin{aligned}
\frac{Q(x, f)}{x} & \leqslant C_{1} \int_{0}^{1}(\log x)^{\operatorname{Re} \tau(\xi)-1} d \xi+\frac{c}{\log x} \\
& =C_{1} \int_{\mathbb{T}}(\log x)^{-2 \sum_{k} \tau_{k} \sin ^{2} \pi k \xi} d \xi+\frac{c}{\log x}=C_{1} I+\frac{c}{\log x} .
\end{aligned}
$$

Оценим интеграл $I$, применяя методы, развитые в [4]. Для этого найдем меру тех $\xi \in \mathbb{T}$, что $2 \sum_{k} \tau_{k} \sin ^{2} \pi k \xi<\varepsilon$ или $\sum_{k} \tau_{k} \cos 2 \pi k \xi>1-\varepsilon, 0<\varepsilon<1$, т.е. меру тех $\xi$, для которых модуль характеристической функции случайной величины $\eta$ принимает "большие" значения. Случайная величина $\eta$ определена так:

$$
\forall k \in \mathbb{Z} \quad P(\eta=k)=\tau_{k}
$$

и, так как $\tau_{k} \geqslant 0, \sum_{k \in \mathbb{Z}} \tau_{k}=1$, то $\tau(\xi)$ можно интерпретировать как характеристическую функцию случайной величины $\eta$. Эта задача рассматривалась в работах [4]-[7]. Мы будем следовать работе [4].

Пусть

$$
E(\varepsilon)=\left\{\xi \in \mathbb{T}: \sum_{k \in \mathbb{Z}} \tau_{k} \cos 2 \pi k \xi \geqslant 1-\varepsilon\right\},
$$

$\operatorname{mes} E$ - мера множества $E$, индуцированная мерой Лебега на $\mathbb{R}$.

Тогда

$$
\begin{aligned}
I & =\int_{\mathbb{T}} \exp \left(\log \log x\left(\sum_{k \in \mathbb{Z}} \tau_{k} \cos 2 \pi k \xi\right)\right) d \xi \\
& =\int_{-1}^{1} \exp (u \log \log x) d \operatorname{mes}\left(\xi \in \mathbb{T}: \sum_{k \in \mathbb{Z}} \tau_{k} \cos 2 \pi k \xi<u\right) \\
& =\int_{-1}^{1} \exp (u \log \log x) d\left(1-\operatorname{mes}\left\{\xi \in \mathbb{T}: \sum_{k \in \mathbb{Z}} \tau_{k} \cos 2 \pi k \xi \geqslant u\right\}\right) \\
& =-\int_{-1}^{1} \exp (u \log \log x) d \operatorname{mes}\left(\xi \in \mathbb{T}: \sum_{k \in \mathbb{Z}} \tau_{k} \cos 2 \pi k \xi \geqslant u\right) .
\end{aligned}
$$


Интегрируя по частям и подставляя пределы интегрирования, получим

$$
I=\frac{1}{\log x}+\log \log x \int_{0}^{2} \exp (\log \log x(1-\varepsilon)) \operatorname{mes} E(\varepsilon) d \varepsilon .
$$

Из (5) видно, что основной вклад в оценку интеграла $I$ дают “малые" значения $\varepsilon$, т.е. важно знать поведение меры тех $\xi$, для которых

$$
\sum_{k \in \mathbb{Z}} \tau_{k} \cos 2 \pi k \xi \geqslant 1-\varepsilon
$$

при "малых" значениях $\varepsilon$.

По равенству Парсеваля получаем

$$
\int_{\mathbb{T}}|\operatorname{Re} \tau(\xi)|^{2} d \xi \leqslant \int_{\mathbb{T}}|\tau(\xi)|^{2} d \xi=\sum_{k \in \mathbb{Z}} \tau_{k}^{2} \leqslant \max _{k} \tau_{k} \sum_{k \in \mathbb{Z}} \tau_{k}=\max _{k} \tau_{k} .
$$

Пусть, далее,

$$
\begin{aligned}
E_{\varphi} & =\left\{\xi \in \mathbb{T}: \sum_{k \in \mathbb{Z}} \tau_{k} \cos 2 \pi k \xi \geqslant \cos \varphi\right\}, \\
2 E_{\varphi} & =E_{\varphi}+E_{\varphi}=\left\{\xi=\xi_{1}+\xi_{2} \in \mathbb{T}: \xi_{1}, \xi_{2} \in E_{\varphi}\right\}, \\
k E_{\varphi} & =(k-1) E_{\varphi}+E_{\varphi} .
\end{aligned}
$$

Если функция $g(\xi)$ положительно определенная, то по теореме Бохнера-Хинчина будет положительно определена и квадратичная форма

$$
\sum_{i, j=1}^{n} g\left(t_{i}-t_{j}\right) z_{i} \overline{z_{j}}
$$

Выберем $t_{1}=0, t_{2}=\xi_{1}, t_{3}=-\xi_{2}$, тогда получим, что положительно определена форма

$$
g(0)\left(\left|z_{1}\right|^{2}+\left|z_{2}\right|^{2}+\left|z_{3}\right|^{2}\right)+g\left(\xi_{1}\right)\left(z_{1} \overline{z_{2}}+\overline{z_{1}} z_{2}\right)+g\left(\xi_{2}\right)\left(z_{1} \overline{z_{3}}+\overline{z_{1}} z_{3}\right),
$$

и по критерию Сильвестра

$$
\left|\begin{array}{ccc}
g(0) & g\left(\xi_{1}\right) & g\left(\xi_{2}\right) \\
g\left(\xi_{1}\right) & g(0) & g\left(\xi_{1}+\xi_{2}\right) \\
g\left(\xi_{2}\right) & g\left(\xi_{1}+\xi_{2}\right) & g(0)
\end{array}\right| \geqslant 0
$$

Если $g(0)=1$, то

$$
\begin{aligned}
& \left|\begin{array}{ccc}
1 & g\left(\xi_{1}\right) & g\left(\xi_{2}\right) \\
g\left(\xi_{1}\right) & 1 & g\left(\xi_{1}+\xi_{2}\right) \\
g\left(\xi_{2}\right) & g\left(\xi_{1}+\xi_{2}\right) & 1
\end{array}\right| \\
& \quad=1+2 g\left(\xi_{1}\right) g\left(\xi_{2}\right) g\left(\xi_{1}+\xi_{2}\right)-g^{2}\left(\xi_{1}\right)-g^{2}\left(\xi_{2}\right)-g^{2}\left(\xi_{1}+\xi_{2}\right) \geqslant 0 .
\end{aligned}
$$


Если обозначить $g\left(\xi_{1}+\xi_{2}\right)=X$, то последнее неравенство перепишется в виде

$$
X^{2}-2 g\left(\xi_{1}\right) g\left(\xi_{2}\right) X+g^{2}\left(\xi_{1}\right)+g^{2}\left(\xi_{2}\right)-1 \leqslant 0 .
$$

Решая это неравенство относительно $X$, получим

$$
g\left(\xi_{1}\right) g\left(\xi_{2}\right)-\sqrt{\left(1-g^{2}\left(\xi_{1}\right)\right)\left(1-g^{2}\left(\xi_{2}\right)\right)} \leqslant X \leqslant g\left(\xi_{1}\right) g\left(\xi_{2}\right)+\sqrt{\left(1-g^{2}\left(\xi_{1}\right)\right)\left(1-g^{2}\left(\xi_{2}\right)\right)} .
$$

Левая часть этого неравенства дает

$$
g\left(\xi_{1}+\xi_{2}\right) \geqslant g\left(\xi_{1}\right) g\left(\xi_{2}\right)-\sqrt{\left(1-g^{2}\left(\xi_{1}\right)\right)\left(1-g^{2}\left(\xi_{2}\right)\right)} .
$$

Данное неравенство выводится также в работе [4]. Если

$$
g\left(\xi_{1}\right) \geqslant \cos \varphi_{1}, \quad g\left(\xi_{2}\right) \geqslant \cos \varphi_{2}, \quad \varphi_{1}, \varphi_{2} \in\left[-\frac{\pi}{2}, \frac{\pi}{2}\right], \quad g(\xi)=\sum_{k \in \mathbb{Z}} \tau_{k} \cos 2 m \pi k \xi,
$$

то $g(\xi)$ - положительно определенная функция и, значит, справедливо следующее соотношение:

$$
g\left(\xi_{1}+\xi_{2}\right) \geqslant g\left(\xi_{1}\right) g\left(\xi_{2}\right)-\sqrt{\left(1-g^{2}\left(\xi_{1}\right)\right)\left(1-g^{2}\left(\xi_{2}\right)\right)} \geqslant \cos \left(\varphi_{1}+\varphi_{2}\right) .
$$

Откуда, для $\xi_{1}, \ldots, \xi_{k}$ таких, что

$$
g\left(\xi_{i}\right) \geqslant \cos \varphi, \quad i=1, \ldots, k
$$

имеем

$$
g\left(\xi_{1}+\cdots+\xi_{k}\right) \geqslant \cos k \varphi .
$$

Для дальнейшего нам понадобится следующий результат из [8].

Teоpema Makbeta-Kheзера. Пусть $A$ и $B$ - множества на mope $\mathbb{T}, A+B=$ $\{c \in \mathbb{T}: c=a+b, a \in A, b \in B\}$. Тогда

$$
\operatorname{mes}(A+B) \geqslant \min (1, \operatorname{mes} A+\operatorname{mes} B) .
$$

Оценим интеграл $\int_{\mathbb{T}}|\operatorname{Re} \tau(\xi)|^{2} d \xi$ снизу. Для подходящего $k$, которое определим позже, будем иметь

$$
\begin{aligned}
& \int_{\mathbb{T}}|\operatorname{Re} \tau(\xi)|^{2} d \xi \\
& \quad=\int_{E_{\varphi}}|\operatorname{Re} \tau(\xi)|^{2} d \xi+\int_{2 E_{\varphi} \backslash E_{\varphi}}|\operatorname{Re} \tau(\xi)|^{2} d \xi+\cdots+\int_{k E_{\varphi} \backslash(k-1) E_{\varphi}}|\operatorname{Re} \tau(\xi)|^{2} d \xi .
\end{aligned}
$$

По теореме Макбета-Кнезера, если неравенство (6) достигается на

$$
\text { mes } A+\operatorname{mes} B \text {, }
$$

то

$$
\operatorname{mes}\left(s E_{\varphi} \backslash(s-1) E_{\varphi}\right) \geqslant \operatorname{mes} E_{\varphi}, \quad 1 \leqslant s \leqslant k,
$$

а тогда

$$
\int_{\mathbb{T}} \operatorname{Re} \tau(\xi) d \xi \geqslant \operatorname{mes} E_{\varphi}(\cos \varphi+\cdots+\cos k \varphi)=\operatorname{mes} E_{\varphi}\left(\frac{k}{2}+\frac{1}{2} \sum_{s=1}^{k} \cos 2 s \varphi\right) .
$$


Теперь найдем наибольшее $k$, для которого справедлива теорема Макбета-Кнезера, т.е. $k$ такое, что $k E_{\varphi} \not \equiv \mathbb{T}$. Для этого очевидно достаточно, чтобы выполнялось неравенство mes $k E_{\varphi}<1$.

Далее, так как $\tau_{0}=\int_{0}^{1} \operatorname{Re} \tau(\xi) d \xi-$ среднее значение $\operatorname{Re} \tau(\xi)$, то ясно, что $\tau_{0} \geqslant$ $\min _{\xi \in \mathbb{T}} \operatorname{Re} \tau(\xi)$. Если $\cos k \varphi>\tau_{0}$, то найдется $\xi^{\prime} \in \mathbb{T}$ такое, что $\tau\left(\xi^{\prime}\right)<\cos k \varphi$ и, следовательно, $\xi^{\prime} \notin k E_{\varphi}$, и тогда использование неравенства (6) законно. Из неравенства $\cos k \varphi>\tau_{0}$ в силу монотонности функции $\cos t$ на отрезке $[0, \pi]$, имеем

$$
k>\frac{\arccos \tau_{0}}{\varphi} .
$$

Пусть $\arccos \tau_{0}=\psi\left(\tau_{0}\right)$, тогда используя неравенство $\cos \psi \geqslant 1-\psi^{2} / 2$, верное при $\psi \in[-\pi / 2, \pi / 2]$, получаем $\tau_{0}=\cos \psi\left(\tau_{0}\right) \geqslant 1-\left(\arccos \tau_{0}\right)^{2} / 2$, откуда $\arccos \tau_{0} \geqslant$ $\sqrt{2\left(1-\tau_{0}\right)}$. Подставим это в $(8)$, получим

$$
k \geqslant \frac{\sqrt{2\left(1-\tau_{0}\right)}}{\varphi} .
$$

Оценим сумму $\sum_{s=1}^{k} \cos 2 s \varphi$. Известно, что

$$
\sum_{s=1}^{k} \cos 2 s \varphi=\cos 2(k+1) \varphi \cdot \frac{\sin k \varphi}{\sin \varphi} .
$$

Так как $\cos k \varphi \geqslant \tau_{0}$, то $k \varphi \leqslant \pi / 2$, и так как $\varphi \in[0, \pi / 2]$, то ядро Дирихле $\sin k \varphi / \sin \varphi \geqslant 0$.

Рассмотрим случай 1 . Пусть $\cos (k+1) \varphi \geqslant-1 / \sqrt{2}$, тогда, подставив в $(10)$, получим

$$
\sum_{s=1}^{k} \cos 2 s \varphi \geqslant-\frac{1}{\sqrt{2}} \frac{\sin k \varphi}{\sin \varphi} \geqslant-\frac{1}{\sqrt{2}} k .
$$

Рассмотрим случай 2. Пусть $\cos (k+1) \varphi<-1 / \sqrt{2}$, тогда $(k+1) \varphi \geqslant 3 / 4 \pi$ и $k \varphi \leqslant \pi / 2$, откуда $\varphi \geqslant \pi / 4$. Используя правую часть (10), получим

$$
\sum_{s=1}^{k} \cos 2 s \varphi=\cos (k+1) \varphi \cdot \frac{\sin k \varphi}{\sin \varphi} \geqslant-\frac{\sin k \varphi}{\sin \varphi} \geqslant-\frac{1}{\sin \frac{\pi}{4}}=-\sqrt{2}
$$

и, так как $-\sqrt{2} \geqslant-k / \sqrt{2}$ для любого $k \geqslant 2$, получаем оценку

$$
\sum_{s=1}^{k} \cos 2 s \varphi \geqslant-\frac{k}{\sqrt{2}}
$$

верную при любом $k \geqslant 2$. Подставив (11) в (7), получим

$$
\int_{\mathbb{T}}|\operatorname{Re} \tau(\xi)|^{2} d \xi \geqslant \operatorname{mes} E_{\varphi} \cdot\left(\frac{k}{2}-\frac{k}{2 \sqrt{2}}\right)=\operatorname{mes} E_{\varphi} \cdot \frac{1}{\sqrt{2}} k .
$$

Собирая оценки (11) и (12), получаем

$$
\max _{k} \tau_{k} \geqslant \operatorname{mes} E_{\varphi} \cdot \frac{1}{\sqrt{2}} k,
$$


и, следовательно, учитывая (9), имеем

$$
\operatorname{mes} E_{\varphi} \leqslant \sqrt{2} \cdot \frac{\max _{k} \tau_{k}}{k} \leqslant \sqrt{2} \cdot \frac{\max _{k} \tau_{k}}{\sqrt{2\left(1-\tau_{0}\right)}} \cdot \varphi .
$$

Оценку сверху для

$$
I_{1}=\frac{1}{\log x} \int_{\mathbb{T}} \exp (\log \log x \cdot \operatorname{Re} \tau(\xi)) d \xi
$$

получим, повторяя вычисления для этого конкретного случая, приведенные в [9].

Вычисления дают такую оценку:

$$
I_{1} \leqslant \frac{\max _{k} \tau_{k}}{\sqrt{1-\tau_{0}}} \cdot \frac{\log \log x}{\log x} \cdot I .
$$

Оценим $I$. Обозначим $u=\sqrt{\log \log x} \sin \varphi$, тогда

$$
\begin{aligned}
I & \leqslant \log x \int_{0}^{\pi / 2} \exp (\log \log x \cdot(-(1-\cos \varphi))) \cos \varphi \sin ^{2} \varphi d \varphi \\
& =\int_{0}^{\pi / 2} \exp \left(-2 \log \log x \sin ^{2} \varphi\right) \cos \varphi \sin ^{2} \varphi d \varphi \\
& =\int_{0}^{\pi / 2} \exp \left(-2 u^{2}\right) \cdot\left(\frac{u}{\sqrt{\log \log x}}\right)^{2} \cdot \frac{d u}{\sqrt{\log \log x}} \\
& \leqslant \frac{\log x}{(\log \log x)^{3 / 2}} \int_{0}^{\infty} \exp \left(-2 u^{2}\right) u^{2} d u \ll \frac{\log x}{(\log \log x)^{3 / 2}} .
\end{aligned}
$$

Подставляя (16) в (15), получаем

$$
I \ll \frac{\max _{k} \tau_{k}}{\sqrt{1-\tau_{0}}} \frac{1}{\sqrt{\log \log x}} .
$$

Наконец, вспоминая, что $Q(x, f) \ll x I$, имеем

$$
Q \ll \frac{\max _{k} \tau_{k}}{\sqrt{1-\tau_{0}}} \frac{x}{\sqrt{\log \log x}} .
$$

Заключение. Представляет интерес использование методов оценки функции концентрации арифметической аддитивной функции, особенно доказательство оценок, аналогичных оценкам, полученным в работах [5], [4].

Основным элементом доказательства теоремы 1 является оценка меры “больших" значений характеристической функции случайной величины, индуцированной арифметической аддитивной функцией $f(n) \in S_{\alpha}$. В общем случае оценки функций концентрации для арифметических аддитивных функций [10], [3] были получены с использованием оценок меры значений характеристической функции из "подходящей" области тора. Тем самым, возникает вопрос об оценке меры и изучении структуры множества значений аргумента характеристической функции, если значение самой характеристической функции взяты из некоторого подмножества тора. Данный вопрос рассматривается в препринте [9]. 


\section{СПИСОК ЦИТИРОВАННОЙ ЛИТЕРАТУРЫ}

[1] G. Halasz, "Über die Mittelwerte multiplikativer zahlentheoretischer Funktionen", Acta Math. Hungar., 19:3-4 (1968), 365-403.

[2] I. Ruzsa, "On the concentration of additive functions", Acta Math. Hungar., 36:3-4 (1980), $215-232$.

[3] Б. В. Левин, А. А. Юдин, “Локальные предельные теоремы для аддитивных арифметических функций", Acta Arith., 22 (1973), 233-247.

[4] I.-M. Deshouillers, G. A. Freiman, A. A. Yudin, "On bounds for the concentration function. I", Structure Theory of Set Addition, Astérisque, 258, Soc. Math. France, Paris, 1999, 425-436.

[5] Т. В. Арак, А. Ю. Зайцев, Равномерные пределъные теоремы для сумм независимых случайных величин, Тр. МИАН, 174, М., Наука, 1986.

[6] Л.П. Постникова, А.А. Юдин, "О функции концентрации", Теория вероятн. и ее примен., 22:2 (1977), 371-375.

[7] А. Г. Постников, А. А. Юдин, "Об оценке функции концентрации суммы одинаково распределенных двумерных целочисленных независимых случайных векторов", Теория вероятн. и ее примен., 26:1 (1981), 156-160.

[8] M. Kneser, "Ein Satz über abelsche Gruppen mit Anwendungen auf die Geometrie der Zahlen", Math. Z., 61:1 (1955), 429-434.

[9] G. A. Freiman, A. A. Yudin, The Interface between Probability Theory and Additive Number Theory (Local Limit Theorems and Structure Theory of Set Addition), Report 2-2003, Tel Aviv University School of Math. Tech., Tel Aviv, 2003.

[10] Б. Б. Петров, Предельные теоремы для сумм независимых случайных величин, Теория вероятн. и матем. стат., 39, Наука, М., 1987.

М. Б. Хрипунова

Поступило

Владимирский филиал Всероссийского заочного

26.12.2005

финансово-экономического института

E-mail: levin@vtsnet.ru

Исправленный вариант

04.04.2007

\section{А. А. Юдин}

Владимирский государственный педагогический университет

E-mail: aayudin@vgpu.vladimir.ru 